\title{
Fixing the Stellar M/L Ratio by Chemospectrophotometric Evolution Models
}

\author{
Marie-Maude de Denus-Baillargeon ${ }^{1}$, Samuel Boissier ${ }^{2}$, \\ Claude Carignan ${ }^{1,3,4}$, Olivier Hernandez ${ }^{1}$ and Philippe Amram ${ }^{2}$ \\ ${ }^{1}$ Département de physique, Université de Montréal \\ C.P. 6128, succ. Centre-Ville, Montréal, H3C 3J7, Canada \\ email: mmaude@astro.umontreal.ca \\ ${ }^{2}$ Laboratoire d'astrophysique de Marseille, Université Aix-Marseille III \\ 38 rue Frederic Joliot-Curie, 13388 Marseille cedex 13, France \\ ${ }^{3}$ Observatoire d'Astrophysique de l'Université de Ouagadougou (ODAUO), \\ UFR/SEA, BP 7021, Ouagadougou 03, Burkina Faso \\ ${ }^{4}$ Department of Astronomy, University of Cape Town, Privite Bag X3, Rondebosch 7701, \\ South Africa
}

\begin{abstract}
The exact contribution of the stellar disk to the overall kinematics of a galaxy remains in most studies a free parameter of the mass models. With the help of chemospectrophotometric evolution models, it is now possible to have a coherent picture of the stellar population of a galaxy including its mass-to-luminosity ratio at every radius spanning a wide range of observable wavelengths. We will focus on discussing the consistency of the mass thus inferred in photometric bands ranging from the FUV to the NIR for individual galaxies and compare this to the maximum-disc hypothesis.
\end{abstract}

Keywords. galaxies: kinematics and dynamics, galaxies: mass-to-light ratio

\section{Introduction}

The number of free-parameters in kinematical analyses of galaxies prevents the unambiguous determination of the dark matter halo parameters and stellar disc mass-toluminosity ratio. Degenerate solutions can make the latter $\left(\Upsilon_{\star}\right)$ vary by up to an order of magnitude and change radically the profile of the halo in the innermost regions, depending heavily on observational as well as conceptual assumptions (Bershady et al. 2010).

Some authors have focussed on the question of the mass of the stellar disk in spiral galaxies, using the baryonic Tully-Fisher relation (Stark, McGaugh \& Swaters, 2009) or chemical evolution along with stellar population synthesis tools to derive color-dependent stellar mass-to-light ratios, (Bell \& de Jong, 2001). Boissier \& Prantzos (1999, 2000) have shown that their multizone chemical evolution models are successful in reproducing the properties of both the local environment of the Galaxy and of nearby galaxies. Since those chemospectrophotometric evolution models supply the photometry in all bands as well as the stellar surface density, the calculation of the $\Upsilon_{\star}$ at all radius follows easily.

\section{Methodology}

The method presented by Boissier \& Prantzos (1999) is a multizone chemical evolution model relying on independently evolving annuli of a galaxy and assuming an inside-out formation. This method calculates the star formation rate as a Schmidt-Kennicutt law 
Table 1. Subsample of SINGS used in this paper. Morphological types are from the NED database and distances are those adopted for the SINGS sample as in the works by Kennicutt et al. (2003) and Gil de Paz et al. (2007)

\begin{tabular}{|c|c|c|c|c|c|}
\hline Name & Distance & Type & Name & Distance & Type \\
\hline NGC 0925 & 9.3 & Sbc & NGC 4321 & 18 & $\mathrm{SAB}(\mathrm{s}) \mathrm{bc}$ \\
\hline NGC 2403 & 3.2 & $\mathrm{SAB}(\mathrm{s}) \mathrm{cd}$ & NGC 4569 & 17 & $\mathrm{SA}(\mathrm{rs}) \mathrm{ab}$ \\
\hline NGC 3 & 16.7 & $\mathrm{SB}(1$ & NGC & 8.2 & $\mathrm{SA}(\mathrm{rs}) \mathrm{bc}$ \\
\hline NGC 3 & 8.3 & $\mathrm{SA}(\mathrm{s}) \mathrm{d}$ & NGC 7793 & 3.9 & $\mathrm{SA}(\mathrm{s}) \mathrm{d}$ \\
\hline NGC 4254 & 17 & $\mathrm{SA}(\mathrm{s}) \mathrm{c}$ & & & \\
\hline
\end{tabular}

depending both on the surface density of the gas and the circular velocity at a given radius. As for the surface density of the gas, a classic exponential disc is assumed as well as a continuous infall of pristine gas varying over time and radius of the galaxy. The resulting photometry profiles in all bands are computed using a KTG93 IMF and the Geneva group spectra. The model was first calibrated on the Milky Way in order to reproduce some quantities observed in the local universe and then a grid of models was created by scaling the original by haloes with different parameters $\lambda$ and $V_{c}$ as prescribed by the $\Lambda$ CDM paradigm (Mo, Mao \& White, 1998) but using pseudo-isothermal haloes. The reader is referred to Boissier \& Prantzos (1999) and Boissier \& Prantzos (2000) for further details and to Muñoz-Matteos et al. (2011) where the results for two different IMFs are presented and compared to the properties of SINGS sample.

From the modeled photometry profiles and stellar surface density it is possible to calculate a modeled $\Upsilon_{\star}$ ratio by using the simple formula:

$$
\Upsilon_{\star}(r)=\frac{\Sigma_{\star \text { model }}(r)}{\Sigma_{\star \text { photo model }}(r)}
$$

It is then straightforward to find the mass of the stellar disk by weighting the mass derived from observed photometry by the $\Upsilon_{\star}$ in the according band :

$$
\Sigma_{\star}=\Upsilon_{\star} \Sigma_{\star \text { photo }}
$$

The $\Sigma_{\star \text { photo }}$ and $\Sigma_{\star \text { photo model }}$ are calculated using the usual photometry $\rightarrow$ surface density conversion:

$$
\begin{array}{r}
\Sigma_{\star \text { photomodel }}=10^{-0.4\left(\mu_{\mathrm{model}}-21.56-\mu_{\odot}\right)} \\
\Sigma_{\star \text { photo }}=10^{-0.4\left(\mu_{\mathrm{observed}}-2.5 \log b / a-21.56-\mu_{\odot}\right)}
\end{array}
$$

where $\mu_{\mathrm{model}}$ and $\mu_{\mathrm{observed}}$ are the modeled and observed surface photometry respectively, and $\mu_{\odot}$ is the solar magnitude in the band. The ROTMOD task of the GIPSY package was used for the conversion from surface density to effective circular velocity. A best-fit of the modeled vs measured photometry profiles was performed by Muñoz-Matteos et al. (2011) to find the most representative model for each galaxy of the SINGS survey. A smaller subsample of those galaxies was selected for which high quality kinematics data was available (table 1), both in $\mathrm{H} \alpha$ and in HI (SINGS and THINGS surveys: Dicaire et al. 2008, Daigle et al. 2006 and Walter et al. 2008).

\section{Results for individual galaxies}

An example of $\Upsilon_{\star}(R)$ found by this method is shown for the widely studied galaxy NGC 3198 in figure 1. Both IMFs are represented here and one can see that the K01 IMF rises steeper and higher in the internal regions. The $\Upsilon_{\star}(r)$ decrease is most pronounced with 


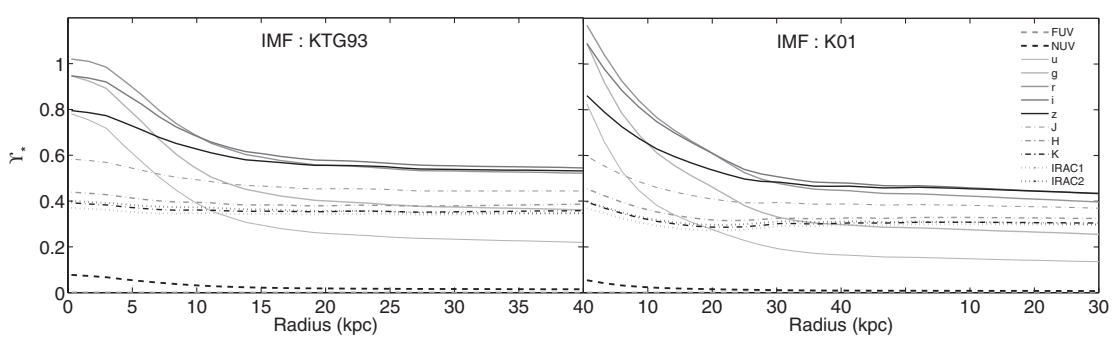

Figure 1. Comparison of the $\Upsilon_{\star}$ in all bands from two different IMFs for the galaxy NGC3198.

radius in the shortest wavelengths whereas it is almost constant in longer wavelengths. We observed the same trend for all galaxies of the sample. This indicates a predominance of evolved stars in the central parts of the galaxy. Figure 2 illustrates that the weighting operation results in a coherent mass of the disc in all bands. Both IMFs perform well but for a slight advantage of K01 particularly in the visible and NIR bands. Therefore we continue our analysis below with only this IMF. Note that the leftmost panel does not show all of the data for the unweighted bands, the UV bands being over three orders of magnitude higher than their visible and IR counterparts. After the correction by $\Upsilon_{\star}$ is applied the UV-bands give results close to the median value. It is, however, the most difficult band to model and the one for which it is less successful: even in the best-fits found by Muñoz-Matteos et al. (2011), models fail to reproduce the UV-bands photometry in several galaxies of their sample, overestimating them systematically in early-types.

From this set of discs, we calculated a median to use it in mass modeling. The highest and lowest velocity considering all bands at each radius were used as an error estimate and represented as a shaded area in the subsequent figures. As figure 3 shows, the level of the contribution of the disc is still far below the rotation curve in the exterior regions of the galaxy. Using this method on the other galaxies of the sample, we find this is also the case with all other galaxies. There is a discrepancy between the effective velocity caused by the stellar disc and the velocity of the interior regions of the actually observed rotation curve for some galaxies of our sample. This problem arises in two different situations: when the galaxy is barred and when a bulge is present. The first case is due to the non-circular motions along the bar, thus lowering or raising the observed rotation curve depending on the strength and orientation of the bar with respect to the major axis of the galaxy (Hernandez et al. (2005)). The second case is obviously caused by an increase of the surface luminosity in the innermost regions where two factors contribute to an increase of the velocities: the $\Upsilon_{\star}$ is at its highest in these particular regions and the effect of flattening the matter distribution in the center causes a rise in the effective velocities compared to a spherical distribution.

From the remaining cases where neither bars nor bulge represent a problem, the effective velocities of the stellar discs are very close to the maximum disc hypothesis. This effect is mainly due to the higher $\Upsilon_{\star}$ found in all bands at small radii for our models. The seeming accordance of our analyses with the maximum-disc hypothesis (see figure 3 ) also has another effect on the mass modeling: by increasing the contribution of the stellar component in the innermost regions of the rotation curve, the method favours a less concentrated dark halo. 


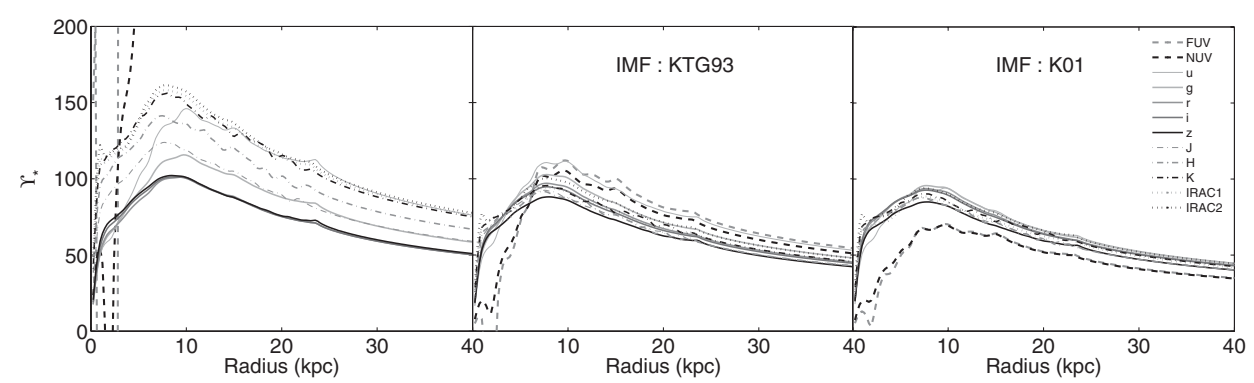

Figure 2. Effective fraction of the rotational velocity caused by the stellar discs as inferred by the photometry in each band. Left: Converted directly from the photometry Middle and right: Converted from photometry and corrected by $\Upsilon_{\star}$
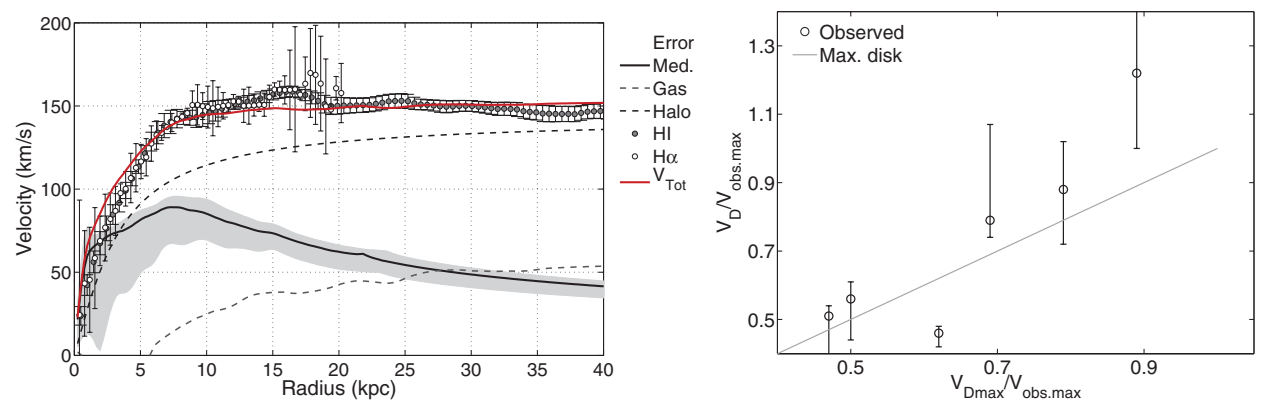

Figure 3. Left: Mass model of NGC3198. The shaded area around the disc's effective velocity represents the error. Right: Accordance of the sample with the maximum-disc hypothesis.

\section{Conclusion}

The method presented here tackles the problem of degeneracy of parameters in mass modeling by applying at all radii a correction based on chemospectrophotometric evolution models to the stellar disc mass inferred by photometry. The discs' masses found by this method are coherent with one another in all bands and they seem to favour the maximum-disc hypothesis and therefore less centrally concentrated haloes.

Even though it gives encouraging first results, this method can still be improved by fine tuning the model on which it is based in order to, among others, reproduce successfully the UV photometry of galaxies.

Future work on this topic will include the results of the mass modeling for all the subsample of SINGS galaxies presented here, as well as assessment of the effects of a bulge and/or a bar if present.

\section{References}

Bell, J. \& de Jong, E., 2001, ApJ, 550, 212

Bershady, M. A., et al. 2010, ApJ 716, 198

Boissier, S. \& Prantzos, N., 1999, MNRAS, 307, 857

Boissier, S. \& Prantzos, N., 2000, MNRAS, 312, 398

Daigle, O., et al. 2006, MNRAS, 367, 469

Dicaire, I., et al. 2008, MNRAS, 385, 553

Gil de Paz, A., et al. 2007 ApJS, 173, 185 
Hernandez, O., et al. 2005, MNRAS, 360, 1201

Kennicutt, R. C., et al. 2003, PASP,115, 928

Mo, H.J., Mao, S., \& White, S.,1998, MNRAS, 295, 319

Muñoz-Matteos, J. C., et al. 2011, In preparation

Stark, D. V., McGaugh, S. S., \& Swaters, R. A. 2009, AJ, 138, 392

Walter, F., et al. 2008, AJ, 136, 2653 